\title{
Modeling the Interplay of Conformational and Electronic Structure in Conjugated Polyelectrolytes
}

\author{
David M. Friday and Nicholas E. Jackson* \\ Department of Chemistry, University of Illinois at Urbana-Champaign, 505 S Mathews \\ Avenue, Urbana, Illinois, 61801, USA \\ E-mail: jacksonn@illinois.edu
}

\begin{abstract}
Conjugated polyelectrolytes (CPEs) combine ionic, electronic, and optical functionality with the mechanical and thermodynamic properties of semiflexible, amphiphilic polyelectrolytes. Critical to CPE design is the coupling between macromolecular conformations, ionic interactions, and electronic transport, the combination of which spans electronic to mesoscopic length scales, rendering coherent theoretical analysis challenging. Here, we utilize a recently developed anisotropic CG model in combination with a phenomenological tight-binding Hamiltonian to explore the interplay of single-chain conformational and electronic structure in CPEs. Accessible single chain conformations are explored as a function of solvent conditions and chain stiffness, reproducing a rich landscape of rod-like, racquet, pearl necklace, and helical conformations observed in previous works. The electronic structure of each conformational archtype is further analyzed, incorporating through-bond coupling, through-space coupling, and electrostatic contributions to the Hamiltonian. Electrostatics is observed to influence electronic structure primarily by modifying the accessible conformational space, and
\end{abstract}


only minimally by direct modulation of on-site energies. Electron transport in CPEs is most efficient in helical and racquet conformations, which is attributed to the flattening of dihedrals and through-space coupling within collapsed conformations. Relatedly, kink formation within racquets does not significantly deteriorate electronic conjugation within CPEs - an insight critical to understanding transport within locally ordered aggregates. These conclusions provide unprecedented computational insight into structure function relationships defining emerging classes of CPEs.

\section{Introduction}

Conjugated polyelectrolytes (CPEs) are a versatile polymer class characterized by $\pi$-conjugated backbones with pendant ionic side chains (Figure 1a). The coupled electronic and ionic functionality characteristic of CPEs has prompted their use in biological, sensing, optical, and electronic applications. ${ }^{1-3}$ In dilute solutions and coacervates, CPEs' conformation dependent optical properties make them effective for the single molecule detection of oppositely charged biomolecules (e.g. DNA, ATP, proteins) and as inks. ${ }^{4,5}$ In semi-dilute or melt states, CPEs are effective mixed conductors and device interlayers, transporting electrons via their $\pi$-electron delocalized conjugated backbones, and ions via their flexible side chains. ${ }^{6}$ This unique combination of functional properties has led to their incorporation into a variety of electronic devices, ${ }^{7-11}$ with emerging interest in biointerfaceable electronic devices where ion/electron transduction is required for sensing and treatment applications. ${ }^{12}$

The physical properties of CPEs arise from a complex interplay of multiscale chemical and morphological features, making CPE design a challenge that could be facilitated by computational modeling. Previous studies of CPEs and doped conjugated polymers have separated the conductivity of ions and electrons, revealing non-monotonic changes in conductivities as a function of dopant concentration, ${ }^{13,14}$ alterations to backbone chemistry, ${ }^{15}$ side chains, ${ }^{16-19}$ solvent,${ }^{20}$ copolymer chemistry, ${ }^{21}$ and multiscale morphology ${ }^{22,23}$ However, due to the significant cost and time requirements for experimental characterizations of CPEs, 
only a small portion of the design space can be explored, and many questions remain regarding the fundamental polymer physics and electronic structure of CPEs. Furthermore, the growing diversity of backbone chemistries, side chains, solvents, salts, dopants, and deposition methods ${ }^{24,25}$ further complicates the exploration of the synthetic and morphological design space. By virtue of its scalability and molecular detail, coarse-grained (CG) molecular modeling ${ }^{26}$ will play an important role in the development of CPEs by identifying the structure-function relationships underlying CPE performance, providing guidance for experimental analysis and high-throughput molecular design.

In conjugated polymers, the $\pi$-conjugated backbone reduces the polymer's flexibility, producing several discrete conformations. Previous computational studies have shown that semi-flexible chains in dilute solutions form coils, toroids, racquets, globules, and cylinders depending on the solvent, temperature, and chain stiffness. ${ }^{27-29}$ In multi-chain systems, simulations show semiflexible and anisotropic polymers can have a variety of ordered structures including cylinders, distorted lamella, and lamella, with head-on or face-on interfacial alignment. ${ }^{30,31}$ In semiconducting conjugated polymers, these different bulk morphologies impact electronic properties substantially, leading to anisotropic charge mobilities. ${ }^{32}$ Theoretical models have shown how nematic alignment in conjugated polymers improves charge carrier mobility. ${ }^{33,34}$ While semiflexible Kremer-Grest CG models can capture the intrinsic stiffness of conjugated polymer backbones, ${ }^{35-37}$ they cannot capture the biaxial nature of anisotropic interactions between $\pi$-conjugated moieties that facilitate the ubiquitous $\pi-\pi$ stacking behavior. Recently, we have developed anisotropically interacting, semiflexible CG models tailored to conjugated polymers, reproducing the hierarchy of semiflexible conformations observed in previous semiflexible polymer simulations, while additionally incorporating $\pi$-stacking and explicitly coupled dihedrals. ${ }^{38}$ These models are particularly efficient for the large, fused-ring $\pi$-conjugated backbone chemistries pervading emerging third and fourth generation conjugated polymer chemistries, ${ }^{39-41}$ and allow for qualitative treatments of electronic structure at the $\mathrm{CG}$ resolution. 
Notwithstanding the complexities induced by semiflexibility and anisotropic monomers, CPE physics is further complicated by the presence of charged moieties, whose electrostatic interactions modify the conformations of polyelectrolytes relative to neutral polymers. This is clearly summarized in the work of Schiessel and Pincus, but will be described briefly here. ${ }^{42}$ If the Bjerrum length of a solution is less than the spacing between charges (e.g. a high dielectric constant), counterions will be dispersed throughout the dilute solution, leading to repulsive electrostatic interactions between charged monomers, resulting in an electrostatically induced chain rigidity. However, when the Bjerrum length exceeds the spacing between charges (e.g. a low dielectric constant), counterions begin to condense onto the chain until the free energy of counterion condensation reaches zero. This counterion condensation reduces the electrostatic repulsion between monomers, and at large Bjerrum length leads to a net attractive interaction between adjacent monomers analogous to dipoleion and dipole-dipole interactions, resulting in globular chain collapse. In the intermediate case of a moderate Bjerrum length, the locally attractive dipole-like electrostatic interactions, combined with a long length scale repulsive electrostatic interactions (due to uncompensated charges), leads to portions of the chain collapsing into globules with stretched chains of monomers between them, a phenomenon described as pearl necklaces. ${ }^{43,44}$ These single-chain conformations have been confirmed experimentally via atomic force microscopy (AFM) and small angle neutron scattering (SANS). ${ }^{45,46}$ In a non-dilute phase, this rich electrostatic interplay between polymer and counterions gives rise to enhanced ion mobility. ${ }^{13}$

The electronic structure of CPEs depends strongly on conformation, in particular the relative orientations of each monomer's $\pi$-system within and between chains. Therefore, understanding and predicting electronic properties of CPEs requires multiscale models capable of simulating mesoscale chain conformations, while simultaneously capturing monomer orientations critical to $\pi$-stacking and charge transport. The electronic coupling between neighboring monomers in a chain depends strongly on the dihedral angle between their $\pi$ systems, ${ }^{47,48}$ while the through space coupling between monomers exhibits a complex depen- 
dence on their relative spatial separation and orientation. The challenge with predicting CPE electronic properties is developing a model that captures the fine level of structural detail required for electronic structure prediction, while being sufficiently computationally scalable to equilibrate systems on morphologically relevant length scales (micrometers). A number of computational approaches have been presented for treating this problem in organic semiconductors (OSCs). One approach for estimating charge mobilities in small molecule OSCs has used all-atom MD simulations to generate a morphology, identifying discrete conjugated segments, parameterizing semi-classical rate theories of charge transport using semi-empirical or density functional theory (DFT) methods, and then solving the master equation to obtain current densities. ${ }^{49}$ To capture the effects of $\pi-\pi$ stacking, dynamic covalent $\pi$-bonds have been implemented in CG MD simulations of polythiophenes, generating cylindrical and lamellar morphologies. ${ }^{31}$ Anisotropic Gay-Berne particles have also been employed to simulate OSCs which were shown to reproduce all-atom radial distribution functions at $\sim 5 \%$ of the all-atom computational cost. ${ }^{50}$ To address the challenge of the large time scales required for polymer relaxation, another approach used soft anisotropic potentials to represent the backbone and side chains, facilitating rapid relaxation, with charge mobility calculations based on predetermined electronic couplings between neighboring monomers. ${ }^{51}$

In this paper we build upon a previous CG modeling work ${ }^{38}$ that captures the anisotropic interactions between $\pi$-conjugated beads via biaxial Gay-Berne (GB) interactions, by including pendant charges and counterions to explore the conformational space of CPEs. The CPE conformations generated are connected to a tight-binding Hamiltonian to predict the adiabatic electronic properties of this system as a function of conformation. First, we introduce the CPE model with its parameters qualitatively linked to poly[3-(6-imidazoliumhexyl)thiophene] bromide (P3ImHTBr), as well as the method used to predict electronic properties. We then simulate the CPE in a variety of solvent conditions resulting in a rich variety of stable, kinetically accessible conformations. Electronic properties of these conformations are then predicted with a full incorporation of the system's electrostatics at a CG resolution. Im- 
portantly, the large spatiotemporal scales accessible via this model let us comment in a statistically meaningful way on the impact that little-studied conformational features such as chain kinks and folds have on the overall electronic structure of CPE aggregates. The results of these simulations are compared to existing experimental works when possible. To the authors' best knowledge, this is the first model that explicitly characterizes the conformations of a CPE in dilute solution as well as the first model to estimate the electronic structure of a CPE from MD-generated conformations using quantum mechanics. These advances provide the first understanding of the coupling between conformation and electronic structure in CPEs as a function of experimentally accessible solvent parameters.

\section{Materials and Methods}

The CG CPE model employed in this work is based on the model introduced by Cohen et al. ${ }^{38}$ with simulation parameters phenomenologically approximating a P3ImHTBr chain ${ }^{36,52-56}$ (see Figure 1). All simulations were run with reduced Lennard-Jones (LJ) units with energy $\epsilon=k_{B} T(\mathrm{~T}=300 \mathrm{~K})$, length $\sigma=0.5 \mathrm{~nm}$, and mass $=40 \mathrm{amu}$. Figure $1 \mathrm{~b}$ shows all types of beads, non-bonding radii, bonds, angles, and dihedrals present in the CG CPE model.

The conjugated thiophene backbone is represented by connected ellipsoidal beads utilizing a biaxial Gay-Berne (GB) potential, as implemented in LAMMPS, ${ }^{57-59}$ to capture the anisotropic intermolecular interactions between non-adjacent thiophene monomers due to $\pi-\pi$ stacking. This potential has been used to describe a variety of anisotropic interactions, including numerous conjugated chemistries. ${ }^{50,60-63}$ The GB well depth in the plane of the ring is set to $\epsilon_{G B}$ while the well depth perpendicular to the ring is set to $3 \epsilon_{G B}$ so that $\pi$ stacking is the energetically most favorable intermolecular orientation (see Figure S1). ${ }^{64,65}$ The dimensions of the GB particle were set to approximate that of a thiophene monomer (See Table S1). Solvent particles are treated implicitly via the use of renormalized intermolecular interaction parameters and an effective dielectric constant - this renders the specific value 

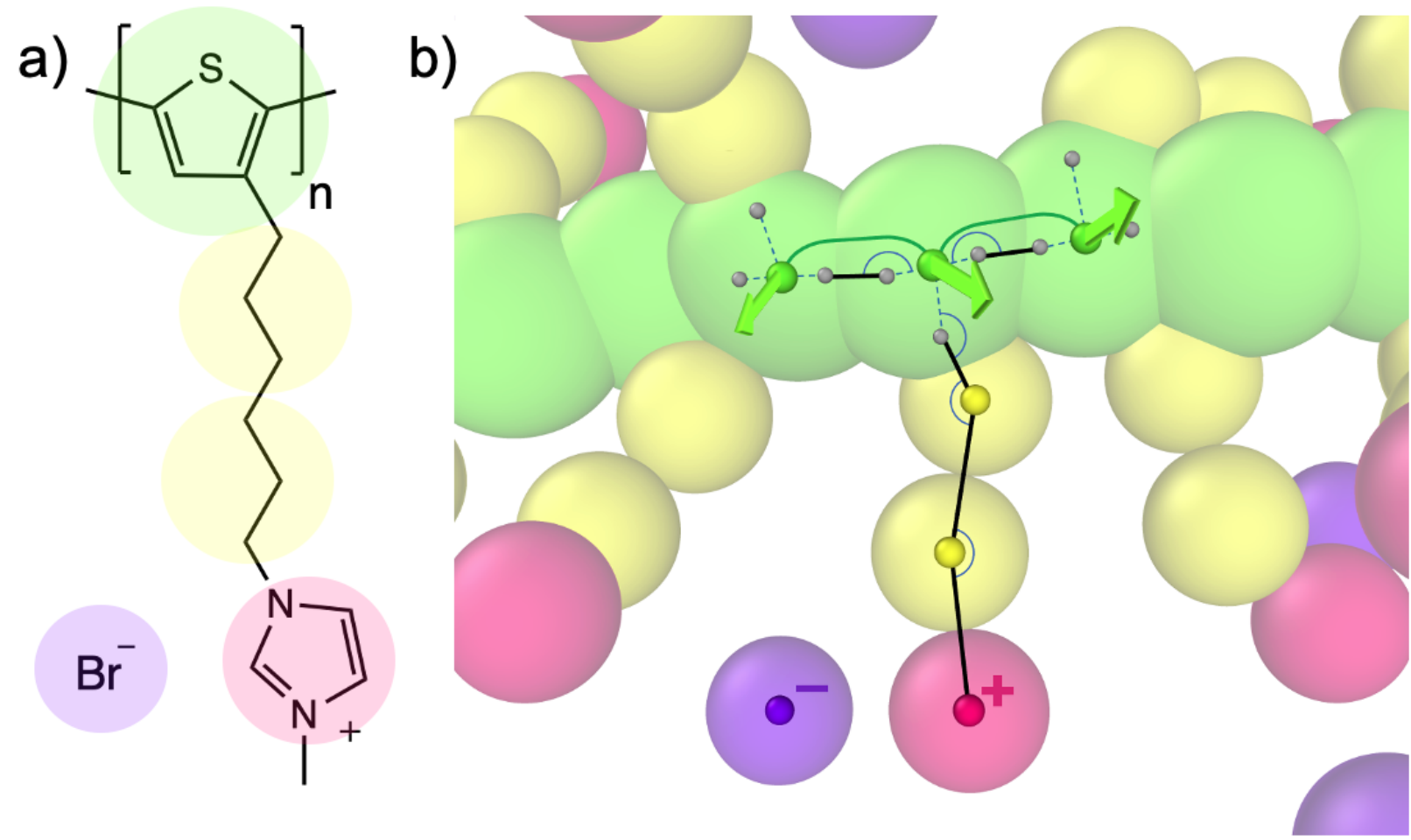

Figure 1: a) Poly[3-(6-imidazoliumhexyl)thiophene] bromide (P3ImHT Br). b) Image of the CG CPE model. Anisotropic back bone beads are green, side chain beads are yellow, cationic pendant charges are pink, anionic counterions are purple, ghost particles are gray, semi-transparent boundaries are LJ/GB radii, and all bonds (black, rigid - dashed), angles (blue), and dihedrals (green) are shown.

of GB well depth $\left(\epsilon_{G B}\right)$ the primary determinant of the solvent quality associated with the conjugated backbone beads. Each backbone bead contains three ghost particles with fixed off-center-of-mass (COM) positions within each ellipsoid that serve as bonding points to adjacent beads. Off-COM bonding points allow for bonded particles to apply a torque to a backbone bead and allows for the incorporation of increased chemical realism within the CG model's molecular geometry. Two-body bond stretching and three-body angle bending interactions between backbone beads were modeled with harmonic potentials, and an OPLSstyle potential ${ }^{66}$ was used to enforce the relative orientations between anisotropic beads. The minimum of the backbone's angle potential $\left(\theta_{0}\right)$ was set to 180 degrees with a prefactor set to $K_{A}=6$ for a 'stiff' chain or $K_{A}=3$ for a 'flexible' chain; these values correspond to a physically reasonable range of persistence lengths for polythiophenes. ${ }^{67}$ The harmonic 
bond stretching constants were set to $2500 \mathrm{kT}$ based on a previous CG P3HT model, and the OPLS-style dihedral force constant $\left(k_{2}\right)$ is $2.4 \mathrm{kT}$ based on the DFT-predicted energy barrier to rotation using a P3HT 14-mer and making the potential symmetric at 0 and 180 degrees. ${ }^{52,68}$ The remaining particles in the system (propyl group, imidazole pendant cations, and bromide counter anions) are represented as single LJ beads. The masses of each particle approximately correspond to the chemical unit they represent. The standard LJ 6-12 potential with a well depth of $0.1 \epsilon$ and diameter of $1.0 \sigma$ was used for non-bonding interactions to describe the side chains as being in good solvent (the $\theta$-condition for homogeneous LJ polymers is $\sim 0.3) .{ }^{69}$ Interactions between LJ and GB beads were modeled as two LJ beads with the same good solvent conditions, and all LJ and GB interactions had a $3 \sigma$ cutoff. All bonded atoms have harmonic bond and angle potentials. An equal number of counterions is added to the system to enforce charge neutrality. Charged particles interact via a short-ranged coulomb potential with a $3 \sigma$ cutoff, and a particle-particle particle-mesh (PPPM) long-range electrostatic solver beyond the cutoff with an accuracy of 1.0E-3. LJ, GB, and Coulomb interactions between bonded beads (including backbone beads) and nextneighbor beads were set to 0 . To further highlight the role of electrostatics in determining CPE conformations, several simulations were run with the charges of all ions set to 0 . These simulations are labeled as having an infinite dielectric. All chains in this work contained 64 monomers. The full list of CG CPE model parameters is provided in Tables S1-S4.

The dynamically accessible conformation space of CPEs in dilute solution is explored throughout a broad parameter space of solvent quality. In all simulations CPE side chains are considered to be in good solvent, and the backbone's $\epsilon_{G B}$ and dielectric constant $(\varepsilon)$ are varied to control solvent quality. All MD simulations were run for $220 \mathrm{k} \tau$ ( 500 ns) using Langevin dynamics (damping parameter equals $1 \tau^{-1}$ ) with a temperature of $300 \mathrm{~K}$ and $\varepsilon$ between 0.1 to 10 in reduced units ( $\sim 11$ to 1100 in real units), in a $65 \times 30 \times 30 \sigma$ box (concentration of $6.5 \mathrm{~g} / \mathrm{L}$ ) with periodic boundary conditions. The time step was set to 0.002 $\tau$. The GB intermolecular potential energy and time correlation function (TCF) of the radius 
of gyration $\left(R_{g}\right)$ were monitored for convergence to equilibrium (for examples, see Figures S3 and S5). Final conformations were identified visually and labeled as a Rod, Pearls, Helix, or Fold. All points in the phase diagrams were the consensus of three independent replicas with different random Langevin seeds: two runs starting from a completely extended state, and one run from a collapsed state (a Helix or a Fold-4 conformation for flexible and stiff chains respectively). The same final conformational state was observed in all simulations except for $K_{A}=6, \epsilon_{G B}=1.2$, and $\varepsilon=1$ in which several 1000k $\tau$-long $(\sim 2 \mu s)$ simulations from both the Fold-1 and Fold-4 conformations failed to converge to the same state, suggesting that the conformations are strongly kinetically trapped under these simulation conditions.

From these simulations, the five distinct conformational states (Rod, Pearls, Helix, Fold1, and Fold-4) were chosen to study the electronic properties of a CPE chain in dilute solution. The five conformations were simulated at several dielectric constants for 110k $\tau$ (250 ns, 10k $\tau$ for equilibration, 100k $\tau$ for sampling). For each simulation $\epsilon_{G B}$ was chosen so that the conformation was stable over the course of the simulation, allowing the electronic properties to be meaningfully averaged and correlated with that conformation. For the Fold states at $\varepsilon=0.1$ or 0.2 , the conformation was stable for at least $60 \mathrm{k} \tau$, though not the entire simulation, which reduced the total averaging. A tight-binding model Hamiltonian was used for electronic analysis. The CPE Hamiltonian is built in a basis of monomer HOMO orbitals $|n\rangle$ and is comprised of three terms: an electrostatic onsite energy $V_{n}$, a throughbond coupling $t_{n, n+1}$, and a through-space coupling $w_{n, m}$ as shown in Equations 1-3. The electrostatic onsite energy estimates the shift in potential energy of each monomer's HOMO due to the electric field generated by the nearby cations and anions. This was calculated after the trajectory was complete by assigning a small additional charge $\left(+10^{-9}\right)$ to each backbone bead, an additional compensating charge to each anion, and then extracting the electrostatic potential experienced by each backbone bead, similar to previous work. ${ }^{54}$ This electrostatic potential was then rescaled to predict the electrostatic energy of a +1 charge localized on that backbone bead. To confirm the accuracy of this approach, the amount 
of added charge was varied from $10^{-1}$ to $10^{-9}$ and revealed that the predicted electrostatic onsite energy became constant after $10^{-6}$ with no indication of decimal precision error down to $10^{-9}$. Note that the energy of the basis orbitals is set to $0 \mathrm{eV}$ in the absence of an electric field for the sake of generality; site energies can be trivially scaled by subtracting the HOMO energy of a thiophene monomer $(\sim-9 \mathrm{eV})$. Typical electrostatic onsite energy values are in the range 0 to $-100 \mathrm{meV}$ for $\epsilon=0.1$ and 0 to $-10 \mathrm{meV}$ for $\epsilon=10$. The through-bond coupling was calculated according to Equation 1 based on a cosine power series of the dihedral angle $(\phi)$ between adjacent monomers. ${ }^{47}$ This equation modulates the coupling from $\sim 1 \mathrm{eV}$ at 0 and 180 degrees to $0 \mathrm{eV}$ at 90 degrees. The through-space coupling between non-bonded monomers was modeled according to Equation 2. This equation qualitatively accounts for the orientation of each monomer $\left(f_{n}\right)$ and the distance between them $\left(r_{n m}\right)$, but does not capture the complex nodal structure of the overlap of monomer orbitals. ${ }^{70}$ Parameters for the through-space coupling are chosen so that the coupling between $\pi$-stacked monomers is $\sim 80 \mathrm{meV}$ at a distance of $1 \sigma$ with a decay constant of $1 \sigma .{ }^{54}$ See Table $\mathrm{S} 4$ for the values of constants in Equations 1 and 2.

$$
\begin{gathered}
t_{n, n+1}=\sum_{i=1}^{5} c_{i} \cos ^{i}\left(\phi_{n, n+1}\right) \\
w_{n m}=J_{\text {inter }}\left(f_{n} \cdot \hat{r}_{n m}\right)^{2}\left(f_{m} \cdot \hat{r}_{n m}\right)^{2}\left(f_{n} \cdot f_{m}\right)^{2} e^{-\alpha\left(\left|r_{n m}\right|-r_{0}\right)}
\end{gathered}
$$

$$
H_{n, m}=\left[\begin{array}{ccccc}
V_{1} & t_{1,2} & w_{1,3} & \ldots & w_{1, N} \\
t_{1,2} & V_{2} & t_{2,3} & \ddots & \vdots \\
w_{1,3} & t_{2,3} & V_{3} & \ddots & w_{N-2, N} \\
\vdots & \ddots & \ddots & \ddots & t_{N-1, N} \\
w_{1, N} & \ldots & w_{N-2, N} & t_{N-1, N} & V_{N}
\end{array}\right]
$$


The tight binding Hamiltonian $\left(H_{n, m}\right.$, Equation 3$)$ is used to calculate the eigenvalues $\left(E_{j}\right)$ and eigenvectors $\left(\Psi_{j}=\sum_{n=1}^{N} c_{n j}|n\rangle\right)$ of the CPE for each snapshot. From each trajectory, the electronic properties were calculated for 1000 snapshots spaced by $1 \mathrm{k} \tau$ to ensure decorrelation of the through-bond and through-space coupling values (see Figures S2 and S4). The Hamiltonian's eigenvalues and eigenvectors were used to calculate the inverse participation ratio (IPR, Equation 4) and the mean and standard deviation of the HOMO density of states (DOS).

$$
I P R_{j}=\left(\sum_{n=1}^{N} c_{n j}^{4}\right)^{-1}
$$

Lastly, to quantify how well the monomers in each conformation are connected to each other in the sense of quantum mechanical charge transport, the Boltzmann weighted electronic communicability (i.e. thermally-averaged Green's function) $G_{n, m}$ between monomers $n$ and $m$ was calculated according to Equation 5 using the partition function from Equation 6 with inverse temperature $\beta .{ }^{71}$ Variations of this Green's function appear in the treatment of non-equilibrium charge transport processes in conjugated systems. ${ }^{72-74}$

$$
\begin{gathered}
G_{n, m}(\beta)=\frac{1}{Z(\beta)} \sum_{j=1}^{N}\left\langle m \mid \Psi_{j}\right\rangle\left\langle\Psi_{j} \mid n\right\rangle e^{-\beta E_{j}} \\
Z(\beta)=\sum_{j=1}^{N} e^{-\beta E_{j}}
\end{gathered}
$$




\section{Results}

A CPE's conformation depends strongly on the solvent effects, which can be usefully decomposed into two contributions arising from short-ranged intermolecular interactions $\left(\epsilon_{G B}\right)$ and the dielectric constant $(\varepsilon)$ associated with long-range charged interactions. ${ }^{42}$ To this end, the conformational phase diagrams for the 'flexible' $\left(K_{A}=3\right)$ and 'stiff' $\left(K_{A}=6\right)$ CPEs were generated by varying the solvent dielectric and the GB well depth $\left(\epsilon_{G B}\right)$, as shown in Figures 2 and 3.

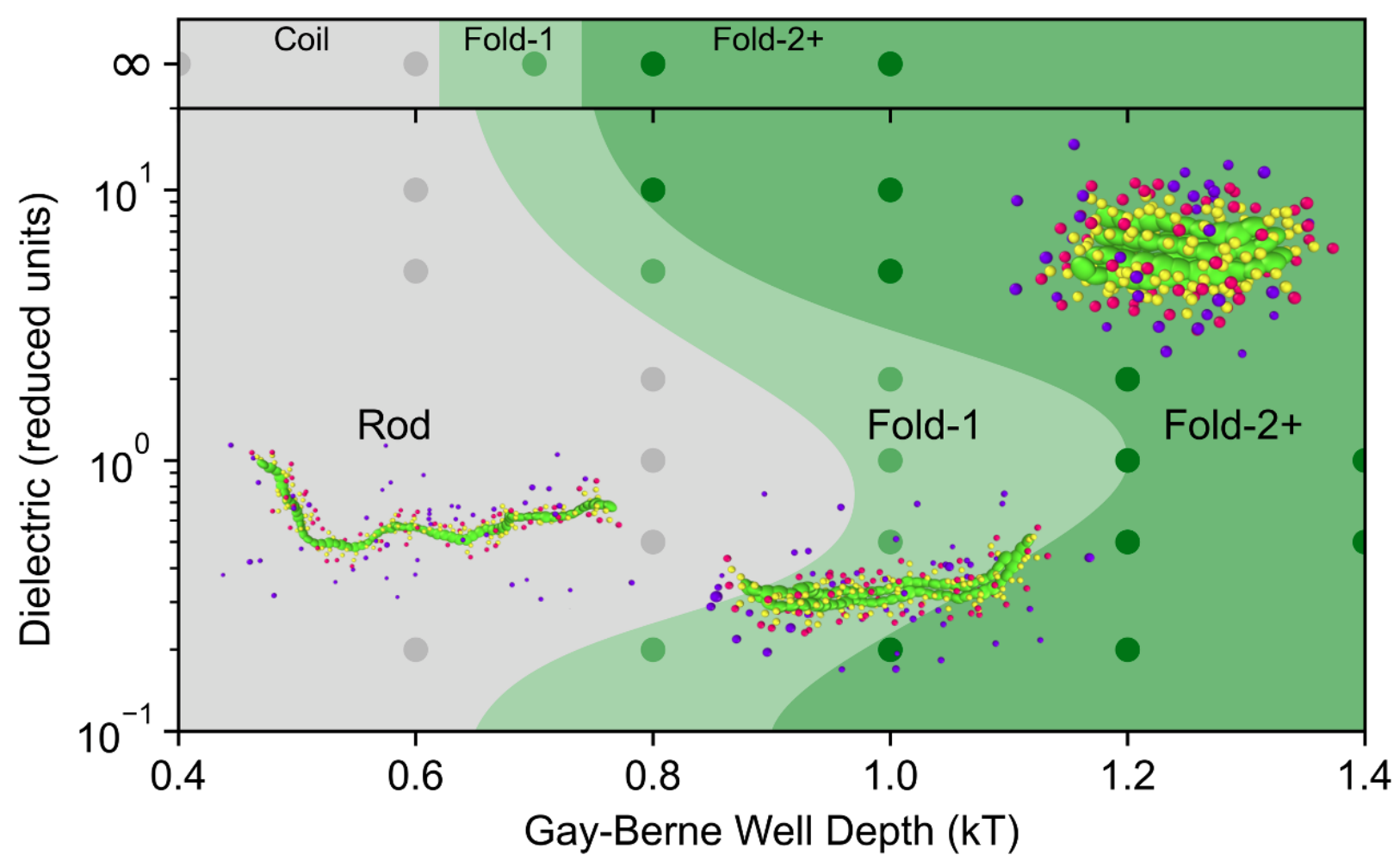

Figure 2: Phase diagram for a flexible chain $\left(K_{A}=6\right)$. Images correspond to representative snapshots of each phase, with the size of all isotropic beads reduced for clarity of the anisotropic backbone beads. Bead coloring is green-backbone, yellow-sidechain, pinkpendant cation, purple-counter anion.

The stiff CPEs proceeded through a series of progressively more folded states, depending on the dielectric, as shown in Figure 2. The transition between a Rod and a single folded state (e.g. Fold-1) was distinct, showing no stable intermediate transition states, and little partial folding or unfolding fluctuations, especially near $\varepsilon=1$ where electrostatic repulsion 


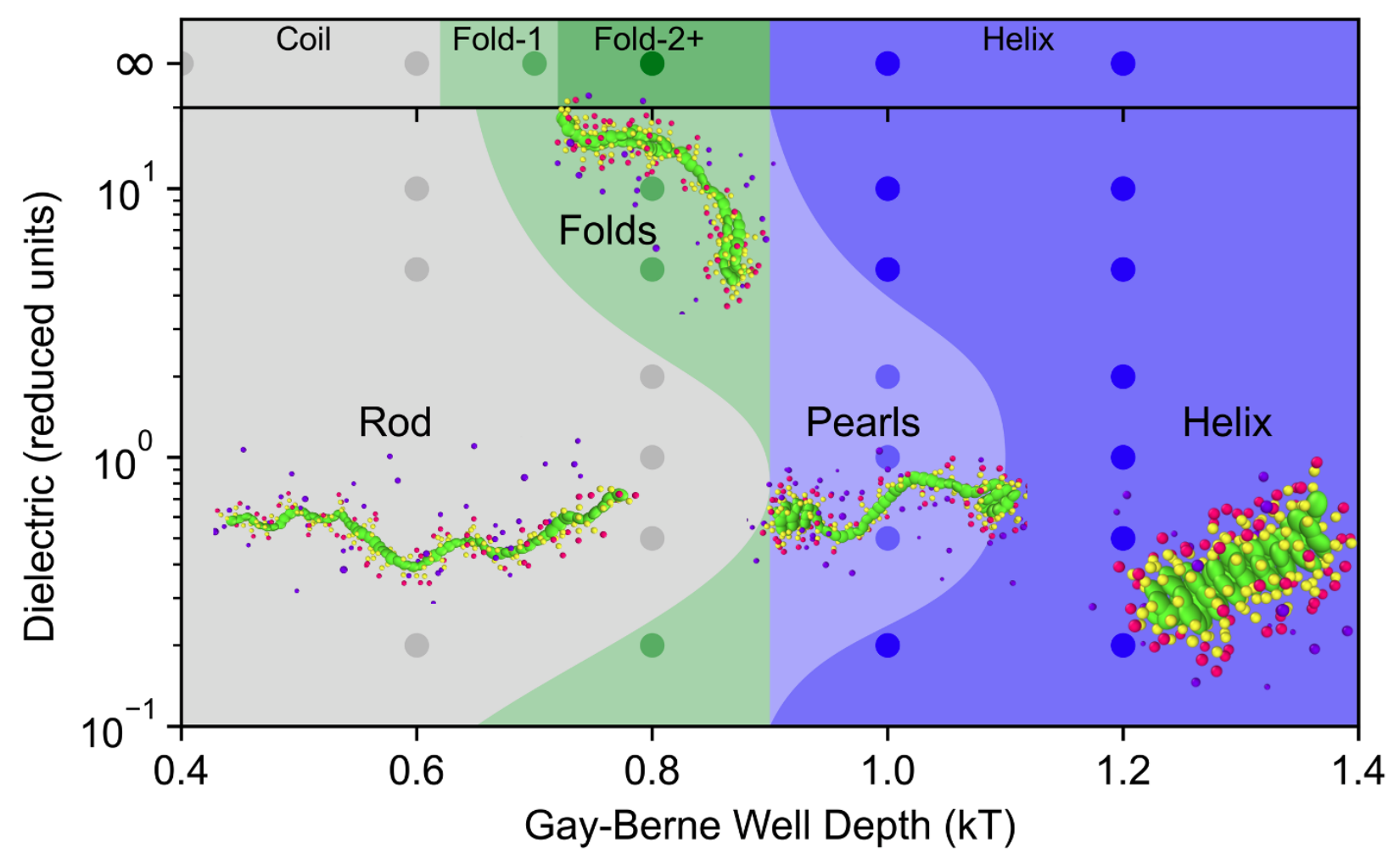

Figure 3: Phase diagram for a flexible chain $\left(K_{A}=3\right)$. Images correspond to representative snapshots of each phase, with the size of all isotropic beads reduced for clarity of the anisotropic backbone beads. Coloring is the same as in Figure 3

was greatest. With a higher $\epsilon_{G B}$, the transitions between successive folds (i.e. Fold-1 to Fold2, or Fold-2 to Fold-3, etc.) became progressively less distinct and more strongly fluctuating, prompting us to only delineate between a Fold-1 and the Fold-2+ conformations in the phase diagram. This process of forming successive folds in semi-flexible polymers has been predicted theoretically as a balance between the gain in binding energy between monomers in the collapsed state and the combined loss of entropy and the energetic cost of forming a kink. ${ }^{27}$ These folded conformations are very similar to racquets ${ }^{27}$ but their racquet head is virtually non-existent due to their flexibility, prompting us to label them as Folds. As described previously, we observed that as the dielectric constant decreases from $\varepsilon=\infty$ to $\varepsilon$ $=1$ (in reduced units), electrostatic repulsion increases along the chain (Figure S6). As the dielectric constant decreased further from $\varepsilon=1$ to $\varepsilon=0.1$, counterion condensation (Figure S7) decreased the electrostatic repulsion, leading to net electrostatic attraction. This change 
in electrostatic repulsion with dielectric led to stretching in both the Rod and Fold-1 states as shown by the change in their radius of gyration $\left(R_{g}\right)$ and asphericity (Figures $\mathrm{S} 8$ ). The transition from increasing electrostatic rigidity to counter-ion condensation occurs at $\varepsilon \sim$ 1 in this model because the Bjerrum length and the spacing between pendant cations are approximately equal $(1 \sigma)$ at this point, making the electrostatic attraction between the counter ions and the $\mathrm{CPE} \sim 1 \mathrm{kT}$.

In contrast to the stiff $\mathrm{CPE}$, as $\epsilon_{G B}$ increased, the flexible CPE first collapsed into pearl-like conformations at $\epsilon_{G B} \leq 1.0$ followed by a single stable Helix conformation for all $\epsilon_{G B} \geq 1.0$. Pearls, resulting from the competition between intra-chain electrostatic repulsion and chain collapse in poor solvent (higher $\epsilon_{G B}$ ), were most stable near $\varepsilon=1$ where electrostatic repulsion was strongest. At higher and lower dielectrics, the chain existed in a dynamic equilibrium with rods, pearls, partial folds, and folds. When pearls were stable at $\epsilon_{G B}=1.0$ (light blue region in Figure 3) they formed helical structures on each end of the chain, whereas when pearls formed at $\epsilon_{G B}=0.8$ they appeared as a single partial fold on each end of the chain, presumably due to the weaker inter-monomer attraction being unable to bend the chain into a spiral. As discussed previously, pearls have been observed experimentally via $\mathrm{AFM}^{45}$ and $\mathrm{SANS},{ }^{46}$ however these experiments were for non-conjugated polymers (typically sulfonated polystyrene) and no internal pearl structure was observed. The internal structure of our pearls resulted from the model's anisotropic potential and awaits experimental validation. For the sake of clarity, further discussion of pearls in this work will refer to the more distinct Pearls conformation with a helical structure $\left(\epsilon_{G B}=1\right.$, $\varepsilon=0.5,1,2)$ denoted as such with a capital $\mathrm{P}$, whereas the more strongly fluctuating folded pearls $\left(\epsilon_{G B}=0.8, \varepsilon=0.2,5,10\right)$ are labeled generically as Folds and are not analyzed further due to their rapid fluctuations between multiple conformations.

As $\epsilon_{G B}$ of the flexible chain increased past the point of pearl conformations, a single Helix conformation was observed for all dielectric constants. This consistency, observed up to $\epsilon_{G B}=2$, likely arises from three causes: 1) each monomer has strong anisotropic GB inter- 
actions ( $\pi$-stacking) with at least two other monomers, 2$)$ monomers have favorable (though much weaker) interactions with monomers on the opposite sides of the Helix, and 3) the side chains' excluded volume promotes twisting. The asphericity of the Helix varied weakly $(<15 \%)$ with dielectric, with the Helix being elongated at dielectric $=1$ due to increased electrostatic repulsion. Kinetic trapping was observed in several simulations either due to an extended CPE forming helical pearls with opposite rotations, resulting in a Helix with a kink in the middle, or the CPE folding briefly and then spiraling, forming a double Helix with a kink on one end. However, over simulation time, the kinks were often equilibrated out of the final CPE conformation. This Helix conformation is similar in appearance to toroid structures described previously but is distinct because it has little free volume inside the Helix and toroids were only observed for much stiffer semiflexible polymers (e.g. $\left.K_{A}>12\right)$. ${ }^{38,75}$ Helices have been observed previously in neutral phenylene ethynylene oligomers and the conformation was also attributed to the presence of strong $\pi$-stacking interactions. ${ }^{76}$

We briefly investigated the impact side chains have on conformations by removing the side chains and transferring the +1 charge to each backbone bead, producing three main observations. First, the flexible chain failed to form helical structures, forming pearls with disordered globular structure and flattened helixes at $\epsilon_{G B}=1.2$ (see Figure S9). This presumably arises from the side chains no longer encouraging tighter rotation via excluded volume. Second, with the +1 charge on the backbone, the counterions were often drawn inside the collapsed chain intercalating themselves between Folds and Helices and increasing the electrostatic potential disorder by a factor of 2-3 (See Figure S6)). Intercalation was never observed in simulations with side chains. Lastly, the through-bond coupling changed for the Rod, Fold-1, and Helix conformations (Pearls and Fold-4 were not comparable without side chains). Rods without side chains showed a small decrease in torsional disorder when compared to rods with side chains, in agreement with previous work which concluded that pendant ionic side chains induce additional torsional disorder in polythiophenes. ${ }^{77}$ However, the Fold-1 and Helix conformations without side chains showed a decrease in through-bond 
coupling. This effect is likely due to coulombic interactions competing with GB interactions, decreasing the dihedral order typically produced by parallel chains as seen in Figure S11.

In a separate set of simulations, side chains were symmetrically attached to every other backbone bead, preserving the total number of side chains, to explore the effect of side-chain symmetry. This symmetry prevented the flexible chain from forming the Helix and Pearl conformations, and delayed chain collapse into folded conformations until the GB well depth was $0.2 \mathrm{kT}$ higher than in Figures 2 and 3. This result indicates that adjusting the geometry of solvophilic side chains can enhance the solubility of conjugated polymers. These results highlight the CG model's ability to explore chemical space to rapidly predict conformations of CPEs and navigate the complex CPE design space.

From the phase diagrams of CPEs (Figures 2 and 3), we selected a set of common singlepolymer conformations (Rod, Fold-1, Fold-4, Pearl, and Helix), for which electronic properties were subsequently investigated. Each conformation was re-simulated starting from a representative snap-shot at several dielectric constants as described previously. Snapshots of the resulting trajectories were analyzed and the eigenvalues and eigenvectors were calculated to estimate the inverse participation ratio (IPR), density of states (DOS), and positions of the HOMO as a function of CPE conformation.

The trends in IPR (Figure 4) and DOS (Figure 5) of each conformation can be understood by the relative contributions from each term of the Hamiltonian. The largest values in the model Hamiltonian (by a factor of 10) are the through-bond coupling energies $\left(t_{n, n+1}\right)$ with couplings of up to $\sim 1 \mathrm{eV}$. Therefore, CPE conformations that planarize dihedral angles and restrict their motion create the strongest electronic connections along the chain. The average through-bond coupling increased substantially as the chain collapsed (see Figure S11), with the Helix conformation having the strongest through-bond coupling, followed by Pearls, Fold-4, Fold-1, and the Rod, respectively. Chain collapse also led to stronger throughspace coupling $\left(w_{n, m}\right)$, making the more collapsed conformations more strongly electronically coupled, increasing the IPR, the average energy of the HOMO (Figure 5), and the spacing 


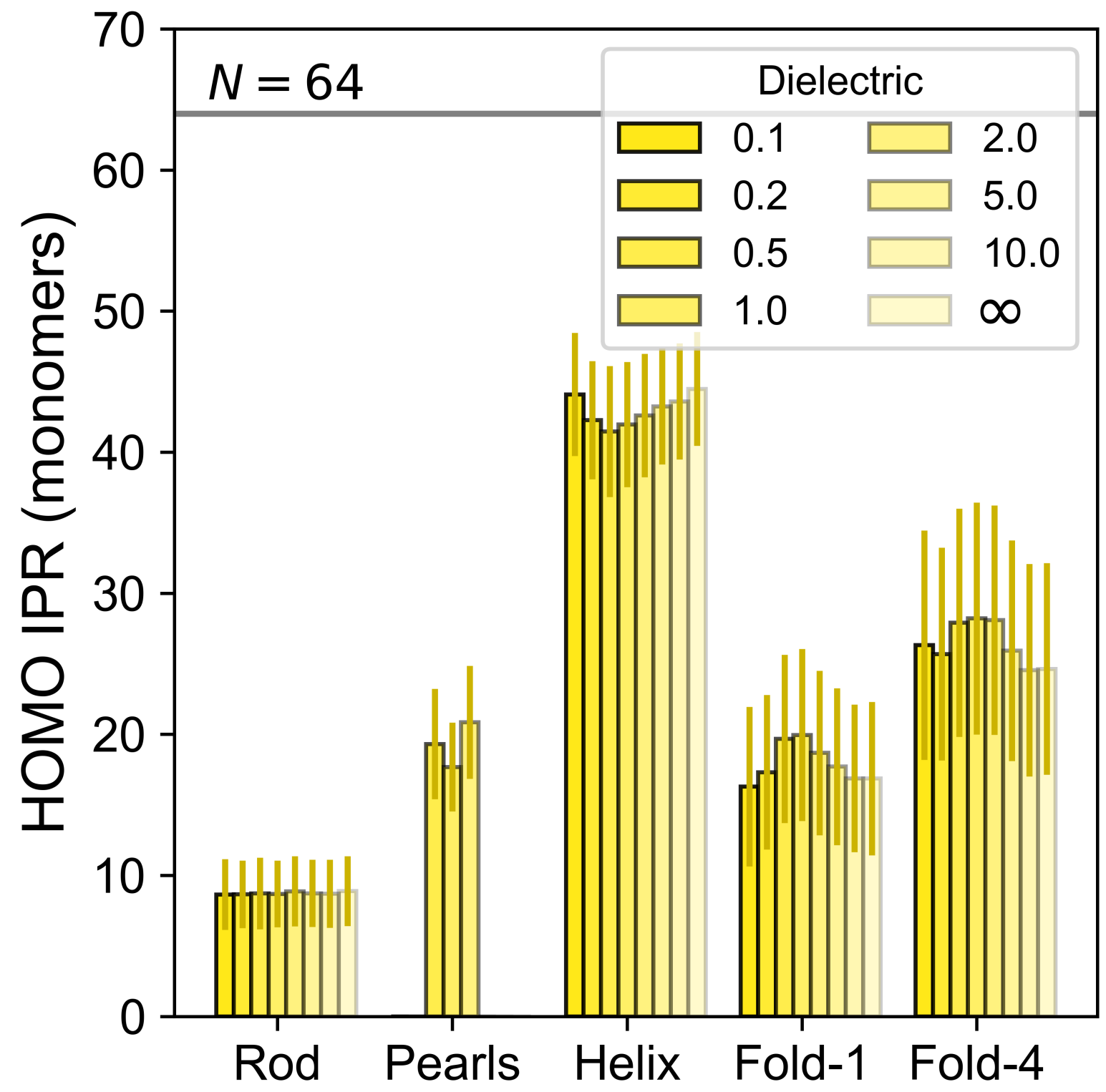

Figure 4: The Inverse Participation Ratio (IPR) for each CPE conformation and dielectric. Error bars represent one standard deviation

between the highest energy MOs (see Figure S12). Lastly, the onsite electrostatic energy $\left(V_{n}\right)$ was observed to play a minor role in modulating the electronic properties of CPEs for most solvent conditions. The small electrostatic disorder (standard deviation of $<30 \mathrm{meV}$ across the chain, see Figure S10) reduces the HOMO IPR by $<2$ monomers (Figure S13), and the HOMO DOS $\sigma$ by $<5 \mathrm{meV}$, although it does reduce the HOMO DOS average by up 

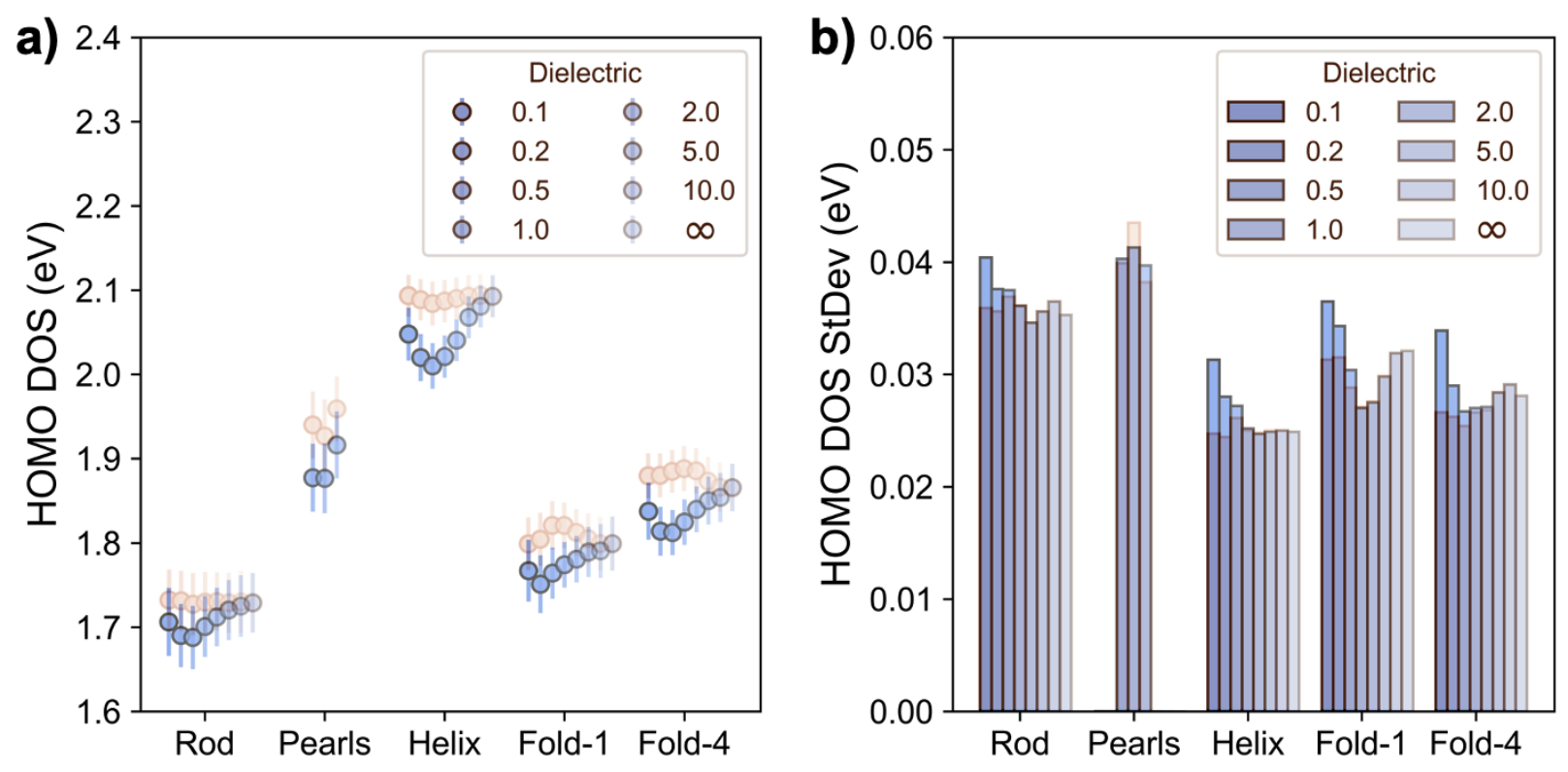

Figure 5: The a) HOMO DOS (average with standard deviations), and b) HOMO standard deviation, for each conformation and dielectric. The orange shadows are results from the same trajectories but with the electrostatic onsite energies $\left(V_{n}\right)$ removed from the Hamiltonian to highlight the role of electrostatics. Error bars represent one standard deviation.

to $0.1 \mathrm{eV}$ when there were a large number of unneutralized pendant ions on the condensed polymers (i.e. at $\varepsilon=1$ ) as shown in Figure 5 .

The IPR and DOS results are displayed pictorially in Figure 6. In the images of electrostatic potential, each conformation has a different value for the average onsite energy (colors), but the standard deviation is consistent across all conformations (Figure S10). The difference in average on-site electrostatic potential determines the drop in the mean of the HOMO DOS (Figure 5a). The images of the HOMO to HOMO-3 (FIgure 6) show the wavefunctions are more delocalized in the collapsed configurations than in the extended configurations, in agreement with the IPR results (Figure 4). Additionally the range in energy gaps between the HOMO and HOMO-3 orbitals is representative of the range of values shown with Figure S12.

The variations in IPR and DOS for $V_{n}=0$ (orange shadows of Figure 5) with dielectric correlate well with the variations in the through-bond coupling for all conformations (Figure S11), which is attributed to electrostatic stretching of the chain. Through-bond coupling 


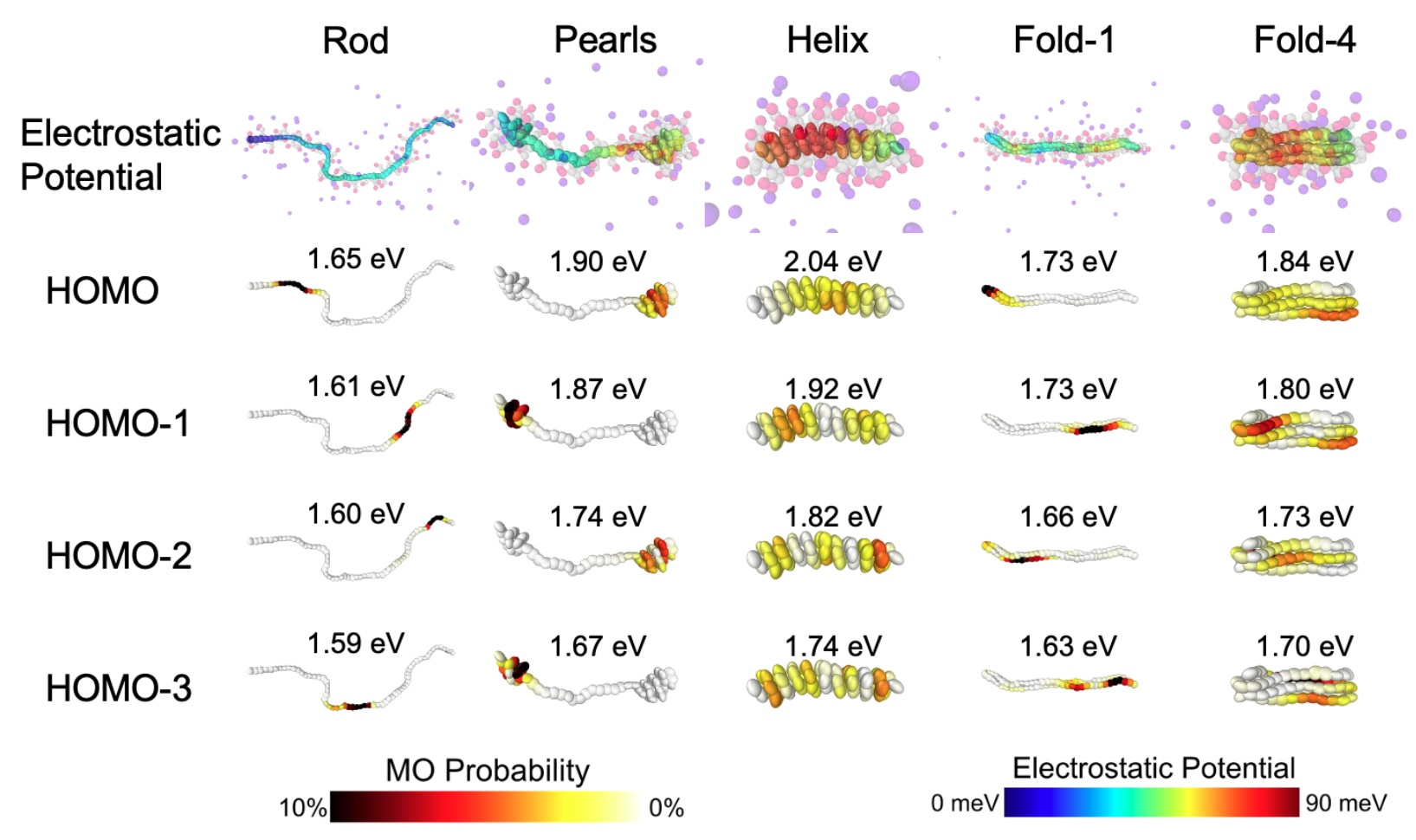

\section{All Simulations are at Dielectric 1}

Figure 6: Example snapshots of each conformation at $\epsilon=1$ showing the electrostatic potential on each backbone bead and the four highest energy molecular orbitals. Note that the energy values here assume that the HOMO monomer site energy $=0 \mathrm{eV}$ in the absence of electrostatic interactions.

correlates positively with the degree of stretching (e.g. asphericity) for the Fold-1 conformation and negatively for Pearls and Helix conformation (compare Figures S8 and S11). This is attributed to the different effects that electrostatically-induced stretching have on $\mathrm{CPE}$ conformations. For the Helix, stretching along the major axis is parallel to the $\pi$-bonds, weakening the GB interactions and increasing dihedral rotation between monomers, which decreases through-bond and through-space coupling. Similarly, stretching Pearls along the major axis appears to increase the length of the string of monomers between the pearls, which exhibits less restricted dihedral rotation and therefore weaker through-bond coupling. In contrast, stretching along the major axis for the Fold-1 conformation is perpendicular to the $\pi$-bonds, which tightens the binding between the two halves of the chain and restricts dihedral rotation, enhancing through-bond and through-space coupling. The Fold-4 
conformation is likely stretched similarly to the Fold-1; however, multiple folds appears to complicate the simple trend.

The limits of electrostatic disorder's impact on a CPE's electronic properties were probed by two methods: 1) reducing the through-bond energy to $10 \%$ of the normal values (i.e. 0.1 $\mathrm{eV}$ ), and 2) removing the side chains and placing a +1 charge on the backbone beads. Reducing through-bond coupling simulates chains with steric hindrance between backbone monomers, reducing the backbone coupling between neighboring $\pi$-systems. These results reveal that the electrostatic disorder plays a more pronounced role in more weakly coupled systems, reducing the IPR when counterion condensation is high, and increasing the standard deviation of the HOMO's energy as shown in Figure S15. Removing side chains increases counterion contact with the now-charged backbone, increasing the standard deviation of on site energies by up to a factor of 3 at $\varepsilon=1$ (Figure S10), but not enough to strongly modify the electronic properties. Note that this does not imply that electrostatics are unimportant for the electronic properties of a dilute CPEs as the electrostatics critically determine CPE's conformations. Instead we find that the influence of electrostatics on electronic structure is primarily due to changing conformations and stretching the CPE, modifying the $\pi$-stacking, rather than the direct modulation of the site energies.

Several of the CPE conformation's average electronic properties (MO energies and wavefunctions, see Figure S12) were found to agree with simple 1D particle-in-a-box intuition. The Rod has the most disordered through-bond coupling and no through space coupling, generating localized lower energy HOMOs that are close in energy, similar to that of a particle-in-a-box with a fluctuating potential energy landscape. On the other extreme, the Helix is strongly coupled with through-bond and through-space interactions, causing the HOMO through HOMO-3 wavefunctions to have evenly spaced nodes, resembling the particle-in-a-box wave functions, with larger energy gaps between successive MOs. The Pearl can be thought of as a particle in a double well potential with nearly degenerate HOMO and HOMO-1 orbitals on each pearl. The Fold-1 is comparable to the Rod with two pri- 
mary differences. First, the adjacent chain reduces the fluctuations in the dihedral angle and creates through-space coupling, which decrease the fluctuations in the potential energy landscape. Second, the kink in the middle of the chain makes it less likely for monomers in the region to participate in the HOMO; however, this effect is small and the kink does not induce disorder significantly beyond the random fluctuations observed in the Rod as shown in Figure 7, and in agreement with atomistic simulations. ${ }^{78}$ In contrast, the Fold-4 has a more complex electronic structure due to through space coupling between non-adjacent parts of the chain. This result highlights the power of this simple CG CPE model in being able to predict electronic properties that agree with intuition in simple cases, but can still predict more complex properties of other physically relevant conformations.

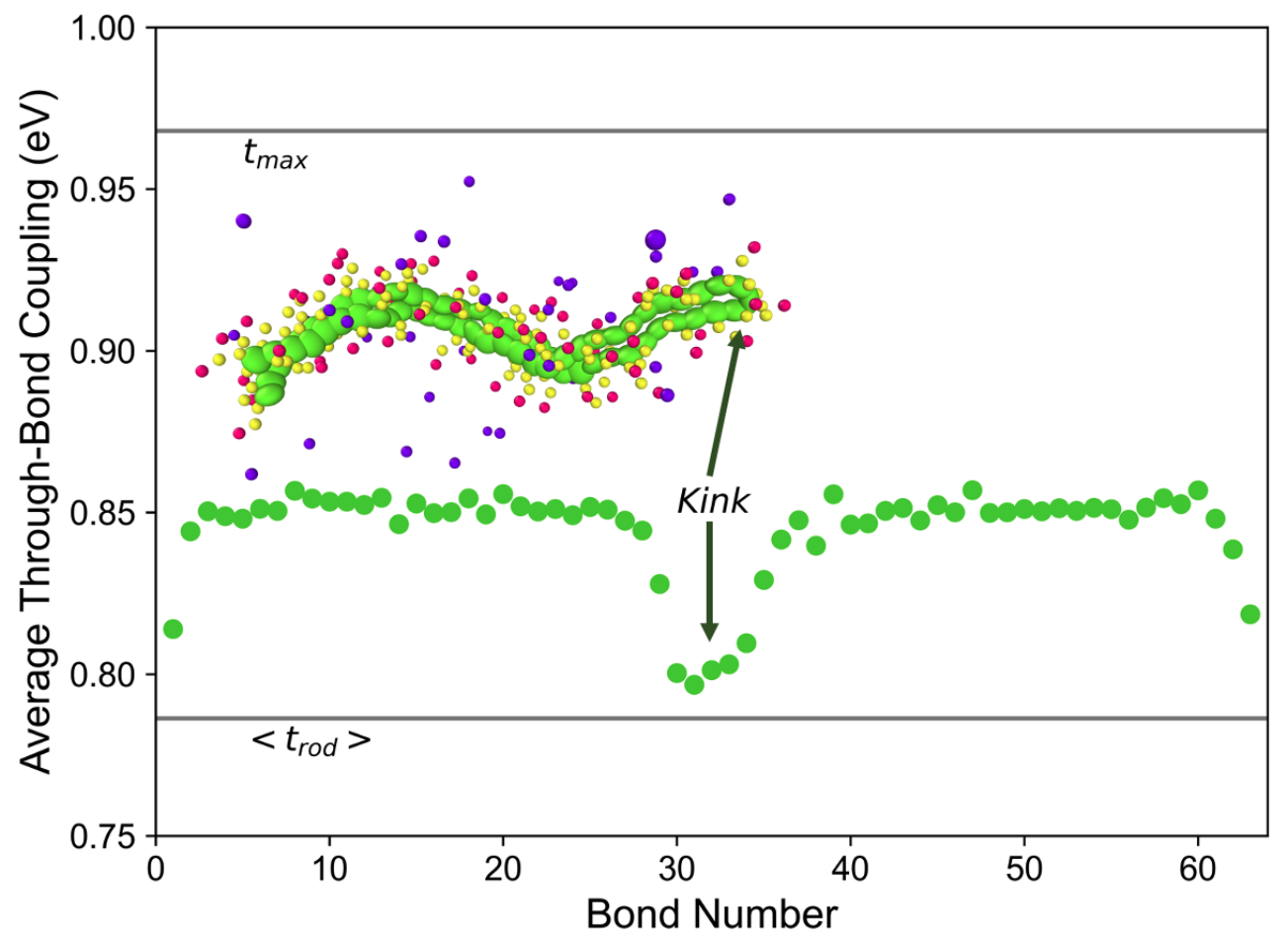

Figure 7: The average through-bond coupling $\left(t_{n, n+1}\right)$ between successive pairs of monomers in a Fold-1 chain. The gray lines correspond to the maximum possible coupling $\left(t_{\max }\right)$ and the average coupling between monomers in a $\operatorname{Rod}\left(\left\langle t_{\text {rod }}\right\rangle\right.$.)

As a simple means to describe the electronic interconnectivity of each conformation, the minimum average electronic communicability between pairs of monomers was calculated (Figure 8). The electronic communicability (Eq 5) represents a Boltzmann weighted aver- 
age of the product of any two monomer's contribution to each wavefunction, derived from graph theory, and is reminiscent of the Green's Function. Determining the minimum average electronic communicability provides an estimate for the conductivity between opposite ends of each CPE conformation. The results clearly show that the expected conductivity of the collapsed conformations (Helix and Fold-4) are greater than the partially collapsed conformations (Pearls and Fold-1) and much greater than the extended Rod conformation. Interestingly, the Pearls electrical connectivity was greater than that of the Fold-1 despite the dihedrals along the string of monomers connecting the two pearls being unconstrained by other monomers as observed in the Fold-1. This result is analogous to predicting the electronic connection between crystalline domains through amorphous regions, and may be able to verify the mechanisms that reduce charge mobility when certain conjugated polymers become hydrated. ${ }^{23}$

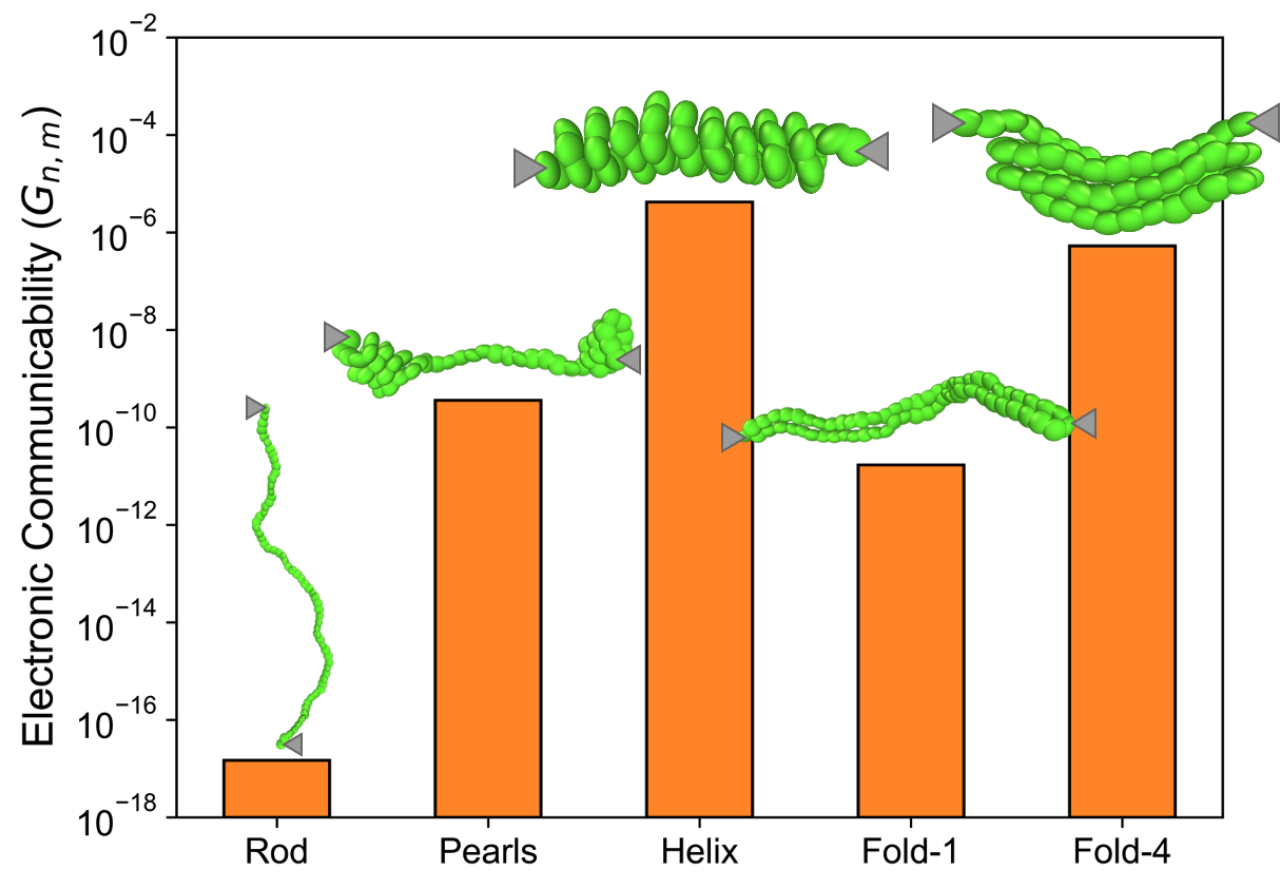

Figure 8: The minimum average electronic communicability (Eq 5) between any pair of monomers in each configuration, which corresponds to the electronic connectivity between opposite ends of the chains. In each case the minimum value was between the first and last monomer in the chain, except for the Fold-1 conformation which had a minimum value between the 32nd monomer and the last. 


\section{Discussion}

The CG CPE model predicts several stable conformations and their electronic properties, reproducing known conformational trends without requiring all-atom simulations. Polythiophene fluorescence depends strongly on solvent, ${ }^{79}$ with poor solvents increasing conjugation lengths by restricting inter-monomer dihedral angles, ${ }^{80}$ and reducing the number of fluorescent sites by increasing energy transfer, funneling the excitation to a single low-energy emitting site. ${ }^{81}$ Our results agree with these results and hypotheses, showing an increase in the average through-bond coupling, IPR, and electronic connectivity as the CPE collapses from a Rod to a Helix or Fold-4 in poor solvent. Though our Hamiltonian is meant for hole transport, the similar form of a Frenkel exciton Hamiltonian means that qualitatively similar conclusions should be expected. Furthermore, atomistic simulations of polythiophenes in good solvent have predicted CPE hole localization lengths of $\sim 7$ monomers and torsional disorder induced by the fluctuating side chains, ${ }^{82}$ both of which compared well with the calculated IPRs (Fig 4), dihedral distributions (Figure S9), and through-bond coupling values (Figure S11) produced by CG CPE simulations.

While this work was loosely based on modeling a thiophene CPE, it can represent a variety of CPEs and has several advantages over CG models with only isotropic interactions. Representing the $\pi$-stacking interactions with a GB potential instead of several rigidly bound LJ beads generates a smooth interaction potential, eliminating potentially noisy interactions with discrete LJ particles. Ellipsoidal particles also deconvolute backbone rotation from sidechain movement, providing true rotation angles between the backbone beads as opposed to the dihedral angle formed by the two side chains on adjacent isotropic backbone beads. ${ }^{52}$ The important intermonomer bond vibrations, angle bending, and dihedral rotations can also be captured with atomistic resolutions via ghost particles within each GB bead, without the need for calculating all intra-ring forces. These carefully preserved degrees of freedom make it possible to independently study the impact CPE structure (i.e. rotational flexibility, semiflexibility, side chain length, ion size, etc.) have on CPE properties, without introducing 
atomistic artifacts, ${ }^{16}$ allowing one to derive general physical insights. The model presented here can also be further modified by incorporating other more complex model Hamiltonians, Frenkel exciton models to predict optical properties, ${ }^{83}$ or incorporating other specialized interactions (i.e. hydrogen bonding) sometimes included in isotropic CG models to represent specific chemical systems. ${ }^{84,85}$

Due to the preserved CG degrees of freedom in this model, we predict molecular-level CPE properties previously unexplored by experimental work. This work has revealed several stable single-polymer conformations for flexible CPEs (e.g. Rod, Pearls, and Helix) and stiffer CPEs (e.g. Rod and Folds), which strongly depend on the solvent quality and electrostatic interactions mediated by the dielectric constant. Each of these conformations had distinct electronic properties, with different average HOMO energies, IPRs, and positions. Our analysis of these conformations shines light on hypotheses about kinks at a fold and counterion induced electrostatic disorder, showing that they appear to have a relatively minor impact on the IPR or DOS compared to the increased through-bond and through-space coupling produced by chain collapse. We also explored the role of side chains, showing that the modeled CPEs with no side chains fail to form helical structures, often contain intercalated counterions, and possess increased torsional disorder. These effects are attributed to the absence of side chain excluded volume, electrostatic attraction between the backbone and counterions, and repulsion between backbone beads. Similarly, CPEs with symmetric side chains on every other monomer were explored and showed that the flexible chain was unable to form Helix or Pearl conformations, preferring the folded states instead. The ease of these comparisons highlights the power of this CG model in rapidly predicting how structural properties of CPEs impact morphological and electronic properties.

The unique combination of electrostatic, semi-flexible, and solvent interactions in CPEs has yielded unexpected experimental results, which this model can probe upon further parameterization. For example, the presence of ionic side chains on polythiophene CPEs has been reported to have no impact on persistence length but does alter the fluorescence life- 
times, possibly due to changes in backbone conformation (e.g. syn- vs anti- conformations), changes in torsional fluctuations, and/or counterion condensation. ${ }^{77}$ In contrast, ionizing a small fraction of a single poly(phenylene-vinylene) (PPV) ionomer's side chains prompts a rapid collapse of the polymer and results in a more structured visible absorbance spectrum, generating questions about electronic energy transfer, conjugation lengths, kinks, and twists. ${ }^{86}$ Furthermore, by changing solvent and ionic strength, PPV with sulfonated sidechains exhibits complex conformational and fluorescence properties when exposed to different solvent conditions. ${ }^{87}$ These studies, as well others, ${ }^{81}$ ask important questions about the physical properties of CPEs and their mechanisms, requiring molecular-level insights which can be extracted with this CG model.

The coupling of conformation and electronic properties in this model also provides unique insights into the mechanisms of recent experimental work, creating collaborative opportunities for improved CPE design. Recent experimental work has revealed single-molecule intra-chain charge transport properties of conjugated oligomers. ${ }^{88}$ If these experiments were expanded to CPEs and CPs, this model could be used to interpret how CPE and CP conformations alter the observed changes in charge transport and begin to elucidate how charge transport occurs in bulk systems. In applications involving transport of optical energy within a single molecule, the Pearl conformation shows promise to be able to absorb higher energy light along the stretched and disordered chain between the pearls, which would then be funneled to the lower energy delocalized states in the condensed pearls, which could be coupled to systems of interest. In denser phases, CPEs have been shown to form coacervates with very rapid (<250 fs) exciton energy transfer between donor-acceptor complexes, with simultaneous control of excited state delocalization lengths. ${ }^{89}$ However the conformations that give rise to these observations are not fully understood, indicating that additional optimization of these coacervates may be possible if the morphology of these CPEs could be observed computationally. It is our hope that this model may shed light on how electronic properties of these systems are derived from molecular-level morphologies, and enable design 
improvements to future CPE systems.

While this model effectively captures several of the most important degrees of freedom influencing CPE conformational and electronic properties, there are opportunities to improve the model's accuracy in the future. First, a significant area for improvement is in estimating the inhomogeneous dielectric of an organic compound in water which has been shown to have a substantial impact on morphology. ${ }^{90}$ We observed that reducing the dielectric constant from 0.2 to 0.1 (22 to 11 in real units) led to an average increase in electrostatic binding energy of almost $1 \mathrm{kT}$. Using dielectric mixing rules or a grid-based approach have been effective in capturing this dielectric inhomogeneity in CG polyelectrolyte simulations ${ }^{85}$ and could be coupled to this approach. Secondly, the equation for through-space coupling is a posteriori, and could be replaced with a more rigorously derived electronic coarse graining approach. ${ }^{91}$ DFT calculations show that the electronic coupling between two parallel perylenediimide molecules is dependent on small $(<1 \AA)$ changes in their relative positions. ${ }^{92}$ This positional variability combined with rotational variability makes improving the through-space coupling a particularly challenging opportunity to address. However, the preservation of the backbone's anisotropy in this CG model provides the information needed to predict inter-monomer coupling to a first order approximation, and more precise systemspecific electronic couplings could be developed using a machine learning approach, an area of active research. ${ }^{93,94}$

\section{Conclusion}

We have adapted a recently developed anisotropic CG model for conjugated polymers to study the interplay of conformational and electronic structure in CPEs. By preserving key molecular degrees of freedom (intermonomer dihedrals, $\pi$-stacking, chain semi-flexibility) for conjugated polymers in conjunction with explicit electrostatics, this model facilitates electronic analysis on CPEs at mesoscopic spatiotemporal scales via coupling with phenomeno- 
logical model Hamiltonians and charge transport analysis. While the model explored in this work is parameterized to reproduce general features of P3ImHTBr, the model is entirely general in its formulation and can be more rigorously correlated with underlying all-atom models in future efforts.

Application of the anisotropic CG model to CPEs in this work reveals a rich conformational landscape for single chain CPEs. In agreement with previous work on semiflexible polymers and polyelectrolytes, our model reproduces the known hierarchy of rod, racquet (e.g. folded), pearl, and helix conformations, while including the critical additional degrees of freedom required to describe the coupling between these large scale conformations and the local molecular structure (e.g. $\pi$-stacking) that influences CPE electronic structure. It was generally observed that collapsed conformations (e.g. Folds, Helix) enhance electronic delocalization and improve charge transport, even in the presence of electrostatically induced disorder and chain folds. We note that the majority of collapsed conformations observed in this work exhibited significant ordering and conjugation along the backbone, which may change depending on the specifics of the CPE chemistry being modeled. For realistic values of the through-bond and through-space couplings, electrostatic interactions in CPEs are observed to influence electronic structure primarily by modifying the global conformation as opposed to the direct modulation of on-site energies. Additional analysis shows the critical role that side chain topology plays in influencing the accessible conformations and the resulting electrostatic landscape. Provided the current lack of knowledge regarding the interplay between molecular and mesoscopic descriptors in CPEs, this work provides critical insights into the structure-function relationships underpinning CPE performance in potential materials applications. 


\section{Acknowledgement}

We thank the American Chemical Society Petroleum Research Fund Doctoral New Investigator award for support of this research. We thank Dr. Artem Rumyantsev for a critical reading of the manuscript.

\section{References}

(1) Hollingsworth, W. R.; Williams, V.; Ayzner, A. L. Semiconducting Eggs and Ladders: Understanding Exciton Landscape Formation in Aqueous $\pi$-Conjugated InterPolyelectrolyte Complexes. Macromolecules 2020, 53, 2724-2734.

(2) Johnston, A. R.; Perry, S. L.; Ayzner, A. L. Associative Phase Separation of Aqueous $\pi$-Conjugated Polyelectrolytes Couples Photophysical and Mechanical Properties. Chemistry of Materials 2021, 33, 1116-1129.

(3) Livshits, M. Y.; Yang, J.; Maghsoodi, F.; Scheberl, A.; Greer, S. M.; Khalil, M. I.; Strach, E.; Brown, D.; Stein, B. W.; Reimhult, E., et al. Understanding the Photochemical Properties of Polythiophene Polyelectrolyte Soft Aggregates with Sodium Dodecyl Sulfate for Antimicrobial Activity. ACS Applied Materials $\&$ Interfaces 2021,

(4) So, R. C.; Carreon-Asok, A. C. Molecular design, synthetic strategies, and applications of cationic polythiophenes. Chemical reviews 2019, 119, 11442-11509.

(5) Danielsen, S. P.; Nguyen, T.-Q.; Fredrickson, G. H.; Segalman, R. A. Complexation of a Conjugated Polyelectrolyte and Impact on Optoelectronic Properties. ACS Macro Letters 2019, 8, 88-94.

(6) Danielsen, S. P.; Sanoja, G. E.; McCuskey, S. R.; Hammouda, B.; Bazan, G. C.; Fredrickson, G. H.; Segalman, R. A. Mixed conductive soft solids by electrostatically 
driven network formation of a conjugated polyelectrolyte. Chemistry of Materials 2018, 30, 1417-1426.

(7) Quek, G.; Roehrich, B.; Su, Y.; Sepunaru, L.; Bazan, G. C. Conjugated Polyelectrolytes: Underexplored Materials for Pseudocapacitive Energy Storage. Advanced Materials 2021, 2104206.

(8) Kesters, J.; Ghoos, T.; Penxten, H.; Drijkoningen, J.; Vangerven, T.; Lyons, D. M.; Verreet, B.; Aernouts, T.; Lutsen, L.; Vanderzande, D., et al. Imidazolium-Substituted Polythiophenes as Efficient Electron Transport Materials Improving Photovoltaic Performance. Advanced Energy Materials 2013, 3, 1180-1185.

(9) Chung, J.; Khot, A.; Savoie, B. M.; Boudouris, B. W. 100th Anniversary of Macromolecular Science Viewpoint: Recent advances and opportunities for mixed ion and charge conducting polymers. ACS Macro Letters 2020, 9, 646-655.

(10) Volkov, A. V.; Wijeratne, K.; Mitraka, E.; Ail, U.; Zhao, D.; Tybrandt, K.; Andreasen, J. W.; Berggren, M.; Crispin, X.; Zozoulenko, I. V. Understanding the capacitance of PEDOT: PSS. Advanced Functional Materials 2017, 27, 1700329.

(11) Moser, M.; Hidalgo, T. C.; Surgailis, J.; Gladisch, J.; Ghosh, S.; Sheelamanthula, R.; Thiburce, Q.; Giovannitti, A.; Salleo, A.; Gasparini, N., et al. Side chain redistribution as a strategy to boost organic electrochemical transistor performance and stability. Advanced Materials 2020, 32, 2002748.

(12) Kim, S.-M.; Kim, C.-H.; Kim, Y.; Kim, N.; Lee, W.-J.; Lee, E.-H.; Kim, D.; Park, S.; Lee, K.; Rivnay, J., et al. Influence of PEDOT: PSS crystallinity and composition on electrochemical transistor performance and long-term stability. Nature communications 2018, 9, 1-9.

(13) Rawlings, D.; Lee, D.; Kim, J.; Magdau, I.-B.; Pace, G.; Richardson, P. M.; Thomas, E. M.; Danielsen, S. P.; Tolbert, S. H.; Miller III, T. F., et al. Li+ and Oxidant 
Addition To Control Ionic and Electronic Conduction in Ionic Liquid-Functionalized Conjugated Polymers. Chemistry of Materials 2021, 33, 6464-6474.

(14) Paulsen, B. D.; Frisbie, C. D. Dependence of conductivity on charge density and electrochemical potential in polymer semiconductors gated with ionic liquids. The Journal of Physical Chemistry C 2012, 116, 3132-3141.

(15) Pittelli, S. L.; Gregory, S. A.; Ponder, J. F.; Yee, S. K.; Reynolds, J. R. Inducing planarity in redox-active conjugated polymers with solubilizing 3, 6-dialkoxy-thieno [3, 2-b] thiophenes (DOTTs) for redox and solid-state conductivity applications. Journal of Materials Chemistry C 2020, 8, 7463-7475.

(16) Dong, B. X.; Nowak, C.; Onorato, J. W.; Strzalka, J.; Escobedo, F. A.; Luscombe, C. K.; Nealey, P. F.; Patel, S. N. Influence of side-chain chemistry on structure and ionic conduction characteristics of polythiophene derivatives: a computational and experimental study. Chemistry of Materials 2019, 31, 1418-1429.

(17) Mai, C.-K.; Arai, T.; Liu, X.; Fronk, S. L.; Su, G. M.; Segalman, R. A.; Chabinyc, M. L.; Bazan, G. C. Electrical properties of doped conjugated polyelectrolytes with modulated density of the ionic functionalities. Chemical Communications 2015, 51, 17607-17610.

(18) Collins, S. D.; Mikhnenko, O. V.; Nguyen, T. L.; Rengert, Z. D.; Bazan, G. C.; Woo, H. Y.; Nguyen, T.-Q. Observing ion motion in conjugated polyelectrolytes with Kelvin probe force microscopy. Advanced Electronic Materials 2017, 3, 1700005.

(19) Moia, D.; Giovannitti, A.; Szumska, A. A.; Maria, I. P.; Rezasoltani, E.; Sachs, M.; Schnurr, M.; Barnes, P. R.; McCulloch, I.; Nelson, J. Design and evaluation of conjugated polymers with polar side chains as electrode materials for electrochemical energy storage in aqueous electrolytes. Energy \& Environmental Science 2019, 12, 1349-1357.

(20) Merkle, R.; Gutbrod, P.; Reinold, P.; Katzmaier, M.; Tkachov, R.; Maier, J.; Lud- 
wigs, S. Mixed conductivity of polythiophene-based ionic polymers under controlled conditions. Polymer 2017, 132, 216-226.

(21) Frankenstein, H.; Stein, E.; Stolov, M.; Khristosov, M. K.; Freger, V.; Frey, G. L. Blends of Polymer Semiconductor and Polymer Electrolyte for Mixed Ionic and Electronic Conductivity. Journal of Materials Chemistry C 2021,

(22) Ma, T.; Dong, B. X.; Onorato, J. W.; Niklas, J.; Poluektov, O.; Luscombe, C. K.; Patel, S. N. Correlating conductivity and Seebeck coefficient to doping within crystalline and amorphous domains in poly (3-(methoxyethoxyethoxy) thiophene). Journal of Polymer Science 2021,

(23) Flagg, L. Q.; Bischak, C. G.; Onorato, J. W.; Rashid, R. B.; Luscombe, C. K.; Ginger, D. S. Polymer crystallinity controls water uptake in glycol side-chain polymer organic electrochemical transistors. Journal of the American Chemical Society 2019, $141,4345-4354$.

(24) Xu, Z.; Park, K. S.; Diao, Y. What is the assembly pathway of a conjugated polymer from solution to thin films? Frontiers in Chemistry 2020, 8.

(25) Choudhary, K.; Chen, A. X.; Pitch, G. M.; Runser, R.; Urbina, A.; Dunn, T. J.; Kodur, M.; Kleinschmidt, A. T.; Wang, B. G.; Bunch, J. A., et al. Comparison of the Mechanical Properties of a Conjugated Polymer Deposited Using Spin Coating, Interfacial Spreading, Solution Shearing, and Spray Coating. ACS Applied Materials \& 6 Interfaces 2021,

(26) Dhamankar, S.; Webb, M. A. Chemically specific coarse-graining of polymers: Methods and prospects. Journal of Polymer Science 2021, 59, 2613-2643.

(27) Schnurr, B.; Gittes, F.; MacKintosh, F. Metastable intermediates in the condensation of semiflexible polymers. Physical Review E 2002, 65, 061904. 
(28) Martemyanova, J.; Stukan, M.; Ivanov, V.; Müller, M.; Paul, W.; Binder, K. Dense orientationally ordered states of a single semiflexible macromolecule: An expanded ensemble Monte Carlo simulation. The Journal of chemical physics 2005, 122, 174907.

(29) Stukan, M.; Ivanov, V.; Grosberg, A. Y.; Paul, W.; Binder, K. Chain length dependence of the state diagram of a single stiff-chain macromolecule: Theory and Monte Carlo simulation. The Journal of chemical physics 2003, 118, 3392-3400.

(30) Boehm, B. J.; Nguyen, H. T.; Huang, D. M. The interplay of interfaces, supramolecular assembly, and electronics in organic semiconductors. Journal of Physics: Condensed Matter 2019, 31, 423001.

(31) Rudyak, V. Y.; Gavrilov, A. A.; Guseva, D. V.; Tung, S.-H.; Komarov, P. V. Accounting for $\pi-\pi$ stacking interactions in the mesoscopic models of conjugated polymers. Molecular Systems Design \&J Engineering 2020, 5, 1137-1146.

(32) Lee, M. J.; Gupta, D.; Zhao, N.; Heeney, M.; McCulloch, I.; Sirringhaus, H. Anisotropy of Charge Transport in a Uniaxially Aligned and Chain-Extended, High-Mobility, Conjugated Polymer Semiconductor. Advanced Functional Materials 2011, 21, 932-940.

(33) Rudnicki, P. E.; MacPherson, Q.; Balhorn, L.; Feng, B.; Qin, J.; Salleo, A.; Spakowitz, A. J. Impact of Liquid-Crystalline Chain Alignment on Charge Transport in Conducting Polymers. Macromolecules 2019, 52, 8932-8939.

(34) Zhang, W.; Milner, S. T.; Gomez, E. D. Nematic order imposes molecular weight effect on charge transport in conjugated polymers. ACS central science 2018, 4, 413-421.

(35) Gartner III, T. E.; Jayaraman, A. Modeling and simulations of polymers: a roadmap. Macromolecules 2019, 52, 755-786.

(36) Marsh, H. S.; Jankowski, E.; Jayaraman, A. Controlling the morphology of model conju- 
gated thiophene oligomers through alkyl side chain length, placement, and interactions. Macromolecules 2014, 47, 2736-2747.

(37) Jackson, N. E.; Kohlstedt, K. L.; Savoie, B. M.; Olvera de la Cruz, M.; Schatz, G. C.; Chen, L. X.; Ratner, M. A. Conformational order in aggregates of conjugated polymers. Journal of the American Chemical Society 2015, 137, 6254-6262.

(38) Cohen, A. E.; Jackson, N. E.; de Pablo, J. J. Anisotropic Coarse-Grained Model for Conjugated Polymers: Investigations into Solution Morphologies. Macromolecules 2021, $54,3780-3789$.

(39) Onwubiko, A.; Yue, W.; Jellett, C.; Xiao, M.; Chen, H.-Y.; Ravva, M. K.; Hanifi, D. A.; Knall, A.-C.; Purushothaman, B.; Nikolka, M., et al. Fused electron deficient semiconducting polymers for air stable electron transport. Nature communications 2018, 9, $1-9$.

(40) Zhuang, W.; Wang, S.; Tao, Q.; Ma, W.; Berggren, M.; Fabiano, S.; Zhu, W.; Wang, E. Synthesis and Electronic Properties of Diketopyrrolopyrrole-Based Polymers with and without Ring-Fusion. Macromolecules 2021, 54, 970-980.

(41) Yao, L.; Zhu, D.; Liao, H.; Haseena, S.; Ravva, M. K.; Cong, S.; Lan, L.; Wang, Y.; Li, Z.; Jiang, L., et al. Fused ambipolar aza-isoindigos with NIR absorption. Organic Chemistry Frontiers 2021, 8, 1170-1176.

(42) Schiessel, H.; Pincus, P. Counterion-condensation-induced collapse of highly charged polyelectrolytes. Macromolecules 1998, 31, 7953-7959.

(43) Dobrynin, A. V.; Rubinstein, M.; Obukhov, S. P. Cascade of transitions of polyelectrolytes in poor solvents. Macromolecules 1996, 29, 2974-2979.

(44) Jeon, J.; Dobrynin, A. V. Necklace globule and counterion condensation. Macromolecules 2007, 40, 7695-7706. 
(45) Kiriy, A.; Gorodyska, G.; Minko, S.; Jaeger, W.; Štěpánek, P.; Stamm, M. Cascade of coil-globule conformational transitions of single flexible polyelectrolyte molecules in poor solvent. Journal of the American Chemical Society 2002, 124, 13454-13462.

(46) Spiteri, M.; Williams, C.; Boué, F. Pearl-necklace-like chain conformation of hydrophobic polyelectrolyte: a SANS study of partially sulfonated polystyrene in water. Macromolecules 2007, 40, 6679-6691.

(47) Bombile, J. H.; Janik, M. J.; Milner, S. T. Tight binding model of conformational disorder effects on the optical absorption spectrum of polythiophenes. Physical Chemistry Chemical Physics 2016, 18, 12521-12533, Publisher: The Royal Society of Chemistry.

(48) McMahon, D. P.; Cheung, D. L.; Troisi, A. Why holes and electrons separate so well in polymer/fullerene photovoltaic cells. The Journal of Physical Chemistry Letters 2011, 2, 2737-2741.

(49) Ruhle, V.; Lukyanov, A.; May, F.; Schrader, M.; Vehoff, T.; Kirkpatrick, J.; Baumeier, B.; Andrienko, D. Microscopic simulations of charge transport in disordered organic semiconductors. Journal of chemical theory and computation 2011, 7, $3335-3345$.

(50) Ricci, M.; Roscioni, O. M.; Querciagrossa, L.; Zannoni, C. MOLC. A reversible coarse grained approach using anisotropic beads for the modelling of organic functional materials. Physical Chemistry Chemical Physics 2019, 21, 26195-26211.

(51) Greco, C.; Melnyk, A.; Kremer, K.; Andrienko, D.; Daoulas, K. C. Generic model for lamellar self-assembly in conjugated polymers: linking mesoscopic morphology and charge transport in P3HT. Macromolecules 2019, 52, 968-981.

(52) Schwarz, K. N.; Kee, T. W.; Huang, D. M. Coarse-grained simulations of the solutionphase self-assembly of poly(3-hexylthiophene) nanostructures. Nanoscale 2013, 5, 2017-2027, Publisher: The Royal Society of Chemistry. 
(53) Maerzke, K. A.; Siepmann, J. I. Transferable potentials for phase equilibria- coarsegrain description for linear alkanes. The Journal of Physical Chemistry B 2011, 115, $3452-3465$.

(54) Khot, A.; Savoie, B. M. Top-Down Coarse-Grained Framework for Characterizing Mixed Conducting Polymers. Macromolecules 2021, 54, 4889-4901.

(55) Shelley, J. C.; Shelley, M. Y.; Reeder, R. C.; Bandyopadhyay, S.; Klein, M. L. A Coarse Grain Model for Phospholipid Simulations. The Journal of Physical Chemistry B 2001, $105,4464-4470$.

(56) Li, P.; Song, L. F.; Merz, K. M. Systematic Parameterization of Monovalent Ions Employing the Nonbonded Model. Journal of Chemical Theory and Computation 2015, 11, 1645-1657, PMID: 26574374.

(57) Brown, W. M.; Petersen, M. K.; Plimpton, S. J.; Grest, G. S. Liquid crystal nanodroplets in solution. J. Chem. Phys. 2009, 130, 044901.

(58) Berardi, R.; Fava, C.; Zannoni, C. A Gay-Berne potential for dissimilar biaxial particles. Chemical Physics Letters 1998, 297, 8-14.

(59) Plimpton, S. Fast parallel algorithms for short-range molecular dynamics. Journal of computational physics 1995, 117, 1-19.

(60) Kleppmann, N.; Klapp, S. H. L. A scale-bridging modeling approach for anisotropic organic molecules at patterned semiconductor surfaces. The Journal of Chemical Physics 2015, 142, 064701.

(61) Goto, M.; Takezoe, H.; Ishikawa, K. Carrier transport simulation in a model liquid crystalline system with the biaxial Gay-Berne potential. The Journal of Chemical Physics 2010, 132, 054506. 
(62) Berardi, R.; Orlandi, S.; Zannoni, C. Columnar phases and field induced biaxiality of a Gay-Berne discotic liquid crystal. Phys. Chem. Chem. Phys. 2000, 2, 2933-2942.

(63) Bacchiocchi, C.; Zannoni, C. Directional energy transfer in columnar liquid crystals: A computer-simulation study. Phys. Rev. E 1998, 58, 3237-3244.

(64) Tsuzuki, S.; Honda, K.; Azumi, R. Model chemistry calculations of thiophene dimer interactions: origin of $\pi$-stacking. Journal of the American Chemical Society 2002, 124, 12200-12209.

(65) Golubkov, P. A.; Ren, P. Generalized coarse-grained model based on point multipole and Gay-Berne potentials. The Journal of chemical physics 2006, 125, 064103.

(66) Jorgensen, W. L.; Maxwell, D. S.; Tirado-Rives, J. Development and testing of the OPLS all-atom force field on conformational energetics and properties of organic liquids. Journal of the American Chemical Society 1996, 118, 11225-11236.

(67) Kuei, B.; Gomez, E. D. Chain conformations and phase behavior of conjugated polymers. Soft matter 2017, 13, 49-67.

(68) Darling, S. B.; Sternberg, M. Importance of side chains and backbone length in defect modeling of poly (3-alkylthiophenes). The Journal of Physical Chemistry B 2009, 113, $6215-6218$.

(69) Graessley, W. W.; Hayward, R. C.; Grest, G. S. Excluded-Volume Effects in Polymer Solutions. 2. Comparison of Experimental Results with Numerical Simulation Data. Macromolecules 1999, 32, 3510-3517, Publisher: American Chemical Society.

(70) Bowen, A. S.; Jackson, N. E.; Reid, D. R.; de Pablo, J. J. Structural Correlations and Percolation in Twisted Perylene Diimides Using a Simple Anisotropic Coarse-Grained Model. Journal of Chemical Theory and Computation 2018, 14, 6495-6504, Publisher: American Chemical Society. 
(71) Estrada, E.; Hatano, N. Tight-binding 'dihedral orbitals' approach to electronic communicability in macromolecular chains. Chemical Physics Letters 2007, 449, 216-220.

(72) Maiti, S. K. Effect of localizing groups on quantum transport through single conjugated molecules. Physica B: Condensed Matter 2007, 394, 33-38.

(73) Yoshizawa, K. An orbital rule for electron transport in molecules. Accounts of chemical research 2012, 45, 1612-1621.

(74) Bruque, N. A.; Pandey, R. R.; Lake, R. K. Electron transport through a conjugated molecule with carbon nanotube leads. Physical Review B 2007, 76, 205322.

(75) Huang, W.; Huang, M.; Lei, Q.; Larson, R. G. A simple analytical model for predicting the collapsed state of self-attractive semiflexible polymers. Polymers 2016, 8, 264.

(76) Prince, R. B.; Saven, J. G.; Wolynes, P. G.; Moore, J. S. Cooperative conformational transitions in phenylene ethynylene oligomers: Chain-length dependence. Journal of the American Chemical Society 1999, 121, 3114-3121.

(77) Danielsen, S. P.; Davidson, E. C.; Fredrickson, G. H.; Segalman, R. A. Absence of Electrostatic Rigidity in Conjugated Polyelectrolytes with Pendant Charges. ACS Macro Letters 2019, 8, 1147-1152.

(78) Qin, T.; Troisi, A. Relation between Structure and Electronic Properties of Amorphous MEH-PPV Polymers. Journal of the American Chemical Society 2013, 135, 1124711256, PMID: 23829780.

(79) Leclerc, M. Optical and electrochemical transducers based on functionalized conjugated polymers. Advanced Materials 1999, 11, 1491-1498.

(80) Palacios, R. E.; Barbara, P. F. Single molecule spectroscopy of poly 3-octyl-thiophene (P3OT). Journal of fluorescence 2007, 17, 749-757. 
(81) Vacha, M.; Habuchi, S. Conformation and physics of polymer chains: a single-molecule perspective. NPG Asia Materials 2010, 2, 134-142.

(82) Simine, L.; Rossky, P. J. Relating chromophoric and structural disorder in conjugated polymers. The journal of physical chemistry letters 2017, 8, 1752-1756.

(83) Tozer, O. R.; Barford, W. Intrachain exciton dynamics in conjugated polymer chains in solution. The Journal of chemical physics 2015, 143, 084102.

(84) Jayaraman, A. 100th Anniversary of macromolecular science viewpoint: modeling and simulation of macromolecules with hydrogen bonds: challenges, successes, and opportunities. ACS Macro Letters 2020, 9, 656-665.

(85) Shen, K.-H.; Fan, M.; Hall, L. M. Molecular Dynamics Simulations of Ion-Containing Polymers Using Generic Coarse-Grained Models. Macromolecules 2021, 54, 2031-2052.

(86) Nguyen, T.-Q.; Schwartz, B. J. Ionomeric control of interchain interactions, morphology, and the electronic properties of conjugated polymer solutions and films. The Journal of chemical physics 2002, 116, 8198-8208.

(87) Smith, A. D.; Shen, C. K.-F.; Roberts, S. T.; Helgeson, R.; Schwartz, B. J. Ionic strength and solvent control over the physical structure, electronic properties and superquenching of conjugated polyelectrolytes. Research on Chemical Intermediates 2007, 33, 125142.

(88) Li, B.; Yu, H.; Montoto, E. C.; Liu, Y.; Li, S.; Schwieter, K.; Rodríguez-López, J.; Moore, J. S.; Schroeder, C. M. Intrachain Charge Transport through Conjugated Donor-Acceptor Oligomers. ACS Applied Electronic Materials 2018, 1, 7-12.

(89) Hollingsworth, W. R.; Magnanelli, T. J.; Segura, C.; Young, J. D.; Bragg, A. E.; Ayzner, A. L. Polyion Charge Ratio Determines Transition between Bright and Dark 
Excitons in Donor/Acceptor-Conjugated Polyelectrolyte Complexes. The Journal of Physical Chemistry C 2018, 122, 22280-22293.

(90) Hou, K. J.; Qin, J. Solvation and entropic regimes in ion-containing block copolymers. Macromolecules 2018, 51, 7463-7475.

(91) Jackson, N. E. Coarse-Graining Organic Semiconductors: The Path to Multiscale Design. The Journal of Physical Chemistry B 2020, 125, 485-496.

(92) Vura-Weis, J.; Ratner, M. A.; Wasielewski, M. R. Geometry and Electronic Coupling in Perylenediimide Stacks: Mapping Structure- Charge Transport Relationships. Journal of the American Chemical Society 2010, 132, 1738-1739.

(93) Jackson, N. E.; Bowen, A. S.; De Pablo, J. J. Efficient multiscale optoelectronic prediction for conjugated polymers. Macromolecules 2019, 53, 482-490.

(94) Jackson, N. E.; Bowen, A. S.; Antony, L. W.; Webb, M. A.; Vishwanath, V.; de Pablo, J. J. Electronic structure at coarse-grained resolutions from supervised machine learning. Science advances 2019, 5, eaav1190.

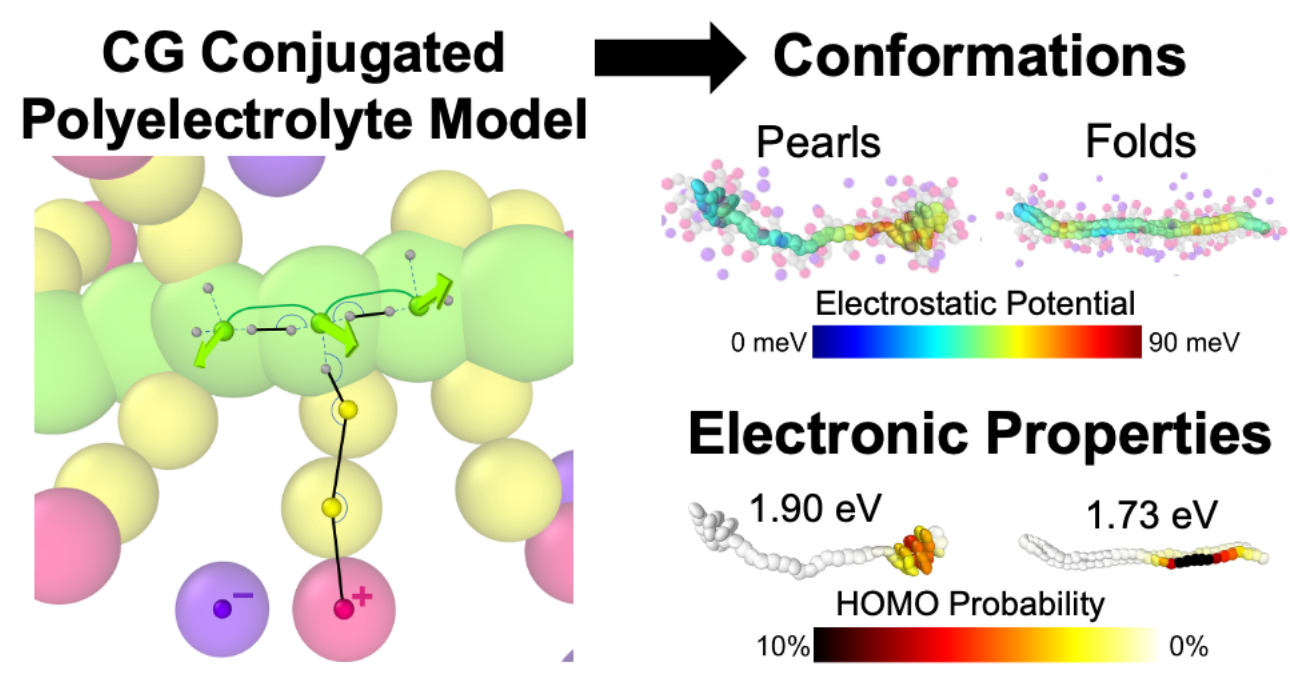

Figure 9: For Table Of Contents Only 